\title{
CONTRACTION OF CYCLIC CODES OVER FINITE CHAIN RINGS
}

\author{
ALEXANDRE FOTUE TABUE AND CHRISTOPHE MOUAHA
}

\begin{abstract}
Aвstract. Let $\mathrm{R}$ be a commutative finite chain ring of invariants $(q, s)$, and $\Gamma(\mathrm{R})$ the Teichmüller's set of $\mathrm{R}$. In this paper, the trace representation cyclic $\mathrm{R}$-linear codes of length $\ell$, is presented, when $\operatorname{gcd}(\ell, q)=1$. We will show that the contractions of some cyclic $R$-linear codes of length $u \ell$ are $\gamma$-constacyclic $R$-linear codes of length $\ell$, where $\gamma \in \Gamma(R) \backslash\left\{0_{R}\right\}$ and the multiplicative order of $\gamma$ is $u$.
\end{abstract}

\section{INTRODUCTION}

Let $\mathrm{R}$ be finite chain ring with invariant $(q, s), \pi: \mathrm{R} \rightarrow \mathbb{F}_{q}$ be the natural ring epimorphism, and $\ell$ a positive integer such that $\operatorname{gcd}(q, \ell)=1$. Let $\mathrm{R}^{\times}$be the group of units of $\mathrm{R}$, and $\gamma \in \mathrm{R}^{\times}$. An $\mathrm{R}$-linear code $\mathscr{C}$ of length $\ell$ is $\gamma$-constacyclic if $\tau_{\gamma}(\mathscr{C})=\mathscr{C}$, where $\tau_{\gamma}: \mathrm{R}^{\ell} \rightarrow \mathrm{R}^{\ell}$, is the $\gamma$-constashift operator, defined by $\tau_{\gamma}\left(\mathbf{c}_{0}, \mathbf{c}_{1}, \cdots, \mathbf{c}_{\ell-1}\right)=\left(\gamma \mathbf{c}_{\ell-1}, \mathbf{c}_{0}, \cdots, \mathbf{c}_{\ell-2}\right)$. Especially, cyclic and negacyclic linear codes correspond to $\gamma=1_{R}$ and $\gamma=-1_{R}$, respectively (see [4]). The residue code of $R$-linear code $\mathscr{C}$ is the $\mathbb{F}_{q}$-linear code $\pi(\mathscr{C}):=\left\{\left(\pi\left(\mathbf{c}_{0}\right), \pi\left(\mathbf{c}_{1}\right), \cdots, \pi\left(\mathbf{c}_{\ell-1}\right)\right):\left(\mathbf{c}_{0}, \mathbf{c}_{1}, \cdots, \mathbf{c}_{\ell-1}\right) \in \mathscr{C}\right\}$. The equality $\pi\left(\tau_{\gamma}(\mathscr{C})\right)=\tau_{\pi(\gamma)}(\pi(\mathscr{C}))$, enables to see that the residue code of any $\gamma$-constacyclic $\mathrm{R}$-linear code, is an $\pi(\gamma)$-constacyclic $\mathbb{F}_{q}$-linear code. In the literature [3, 5, 10, 11, 12, the class of $\gamma$-constacyclic $R$-linear codes, which are studied, have the following property $\gamma \in 1_{R}+R \theta$.

In this paper, on the one hand, we will describe each $\gamma$-constacyclic $R$-linear code of length $\ell$, as contraction of a cyclic $\mathrm{R}$-linear code of length $u \ell$, and on the other hand, we will investigate on the structure of $\gamma$-constacyclic $R$-linear codes, where $\gamma \in \Gamma(R) \backslash\left\{0_{R}\right\}$.

The present paper is organized as follows. In Sect. 2. we present results which will be used in the following sections. Sect. 3 studies the subring subcode and trace code of a linear codes over finite chain rings. In Sect 4 the trace-description of cyclic linear codes over finite chain rings is presented. For any $\gamma \in \Gamma(R)$, we proceed to investigate on the structural properties of $\gamma$-constacyclic codes of arbitrary length $\ell$, in Sect. 5 .

\section{BACKGROUND ON FINITE CHAIN RINGS}

Throughout of this section, $R$ is a commutative ring with identity and $J(R)$ denoted the Jacobson radical of $R$, and $R^{\times}$denotes the multiplicative group of units of $R$. The definitions and results on the finite chain rings are extracted in monographs [6, 8].

Definition 2.1. We say that $\mathrm{R}$ is a finite chain ring of invariants $(q, s)$, if:

(1) $\mathrm{R}$ is local principal ideal ring;

(2) $\mathrm{R} / \mathrm{J}(\mathrm{R}) \simeq \mathbb{F}_{q}$ and $\mathrm{R} \supsetneq \mathrm{R} \theta \supsetneq \cdots \supsetneq \mathrm{R} \theta^{s-1} \supsetneq \mathrm{R} \theta^{s}=\{0\}$, where $\theta$ is a generator of $\mathrm{J}(\mathrm{R})$.

The map $\pi: R \rightarrow \mathbb{F}_{q}$ denotes the canonical projection.

Lemma 1. Let $\mathrm{R}$ be a finite chain ring of invariants $(q, s)$, and $\theta$ be a generator of $\mathrm{J}(\mathrm{R})$. Then

(1) $R^{\times}=R \backslash J(R)$, and the ideals of $R$ are precisely $\mathrm{J}(\mathrm{R})^{t}=\mathrm{R} \theta^{t}$, where $t \in\{0,1, \cdots, s\}$;

(2) $\left|\mathrm{R}^{\times}\right|=q^{(s-1)}(q-1)$ and $\left|\mathrm{J}(\mathrm{R})^{t}\right|=q^{s-t}$, for every $t \in\{0,1, \cdots, s\}$.

Theorem 1. Let $\mathrm{R}$ be a finite chain ring of invariants $(q, s)$, and $\theta$ be a generator of $\mathrm{J}(\mathrm{R})$. Then

2010 Mathematics Subject Classification. 16P10; 65F30; $94 \mathrm{~B} 15$.

Key words and phrases. Linear Codes; Constacyclic Codes; Finite Chain Rings; Trace Map. 
(1) $R^{\times}=\Gamma(R)^{*} \cdot(1+R \theta)$, and $\Gamma(R)^{*} \simeq \mathbb{F}_{q} \backslash\{0\}$ (as multiplicative group) where $\Gamma(R)^{*}:=\{\boldsymbol{b} \in R: \boldsymbol{b} \neq$ $\left.0, \boldsymbol{b}^{q}=\boldsymbol{b}\right\}$

(2) $\Gamma(R)^{*}$ is a cyclic subgroup of $R^{\times}$, of order $q-1$ and $\left|1_{R}+R \theta\right|=q^{s-1}$;

(3) for every element $\boldsymbol{a} \in \mathrm{R}$, there exists a unique $\left(\boldsymbol{a}_{0}, \boldsymbol{a}_{1}, \cdots, \boldsymbol{a}_{s-1}\right) \in \Gamma(\mathrm{R})^{s}$, such that $\boldsymbol{a}=\boldsymbol{a}_{0}+\boldsymbol{a}_{1} \theta+$ $\cdots+\boldsymbol{a}_{s-1} \theta^{s-1}$.

Definition 2.2. Let $\mathrm{R}$ be a finite chain ring of invariants $(q, s)$, and $\theta$ be a generator of $\mathrm{J}(\mathrm{R})$. The set $\Gamma(\mathrm{R})=\Gamma(\mathrm{R})^{*} \cup\{0\}$ is called the Teichmüller set of $\mathrm{R}$.

We say that the ring $S$ is an extension of $R$ and we denote it by $S \mid R$ if $R$ is a subring of $S$ and $1_{R}=1_{S}$. We denote by $\operatorname{rank}_{R}(S)$, the rank of $R$-module $S$. We denote by $\operatorname{Aut}_{R}(S)$, the group of ring automorphisms of $\mathrm{S}$ which fix the elements of $\mathrm{R}$.

Definition 2.3. Let $\mathrm{R}$ be a finite chain ring of invariants $(q, s)$. We say that the finite chain ring $\mathrm{S}$ is the Galois extension of $\mathrm{R}$ of degree $m$, if

(1) $\mathrm{S} \mid \mathrm{R}$ is unramified, i.e. $\mathrm{J}(\mathrm{S})=\mathrm{J}(\mathrm{R}) \mathrm{S}$;

(2) $\mathrm{S} \mid \mathrm{R}$ is normal, i.e. $\mathrm{R}:=\left\{\boldsymbol{a} \in \mathrm{S}: \varrho(\boldsymbol{a})=\boldsymbol{a}\right.$ for all $\left.\varrho \in \mathrm{Aut}_{\mathrm{R}}(\mathrm{S})\right\}$.

Proposition 1. Let $\mathrm{R}$ be a finite chain ring of invariants $(q, s)$. Let $\mathrm{S}$ is the Galois extension of $\mathrm{R}$ of degree $m$. Then

(1) $\mathrm{S}$ is a free $\mathrm{R}$-module of rank $m$;

(2) $\operatorname{Aut}_{\mathrm{R}}(\mathrm{S})$ is cyclic of order $m$;

(3) $\mathrm{S}=\mathrm{R}[\xi]$ where $\xi$ is a generator of $\Gamma(\mathrm{S})$.

Definition 2.4. Let $\mathrm{S} \mid \mathrm{R}$ be the Galois extension of finite chain rings of degree $m$ and $\sigma$ be a generator of $\operatorname{Aut}_{\mathrm{R}}(\mathrm{S})$. The map $\operatorname{Tr}_{\mathrm{R}}^{\mathrm{S}}:=\sum_{i=0}^{m-1} \sigma^{i}$, is called the trace map of the Galois extension $\mathrm{S} \mid \mathrm{R}$.

Proposition 2. 6, Chap. XIV] Let $\mathrm{S} \mid \mathrm{T}$ and $\mathrm{R} \mid \mathrm{T}$ be Galois extensions of finite chain rings. Then

(1) $\mathrm{R}=\left\{\boldsymbol{a} \in \mathrm{S}: \sigma(\boldsymbol{a})=\boldsymbol{a}\right.$ for all $\left.\sigma \in \mathrm{Aut}_{\mathrm{R}}(\mathrm{S})\right\}$;

(2) the bilinear form $\varphi:(\boldsymbol{a}, \boldsymbol{b}) \mapsto \operatorname{Tr}_{\mathrm{R}}^{\mathrm{S}}(\boldsymbol{a b})$ is nondegenerate;

(3) $\operatorname{Tr}_{R}^{\mathrm{S}}$ is a generator of $\mathrm{S}$-module $\operatorname{Hom}_{\mathrm{R}}(\mathrm{S}, \mathrm{R})$, and $\operatorname{Tr}_{\mathrm{T}}^{\mathrm{R}} \circ \operatorname{Tr}_{\mathrm{R}}^{\mathrm{S}}=\operatorname{Tr}_{\mathrm{T}}^{\mathrm{S}}$.

\section{LINEAR CODES OVER FINITE CHAIN RINGS}

Recall that an $\mathrm{R}$-linear code of length $\ell$ is an $\mathrm{R}$-submodule of $\mathrm{R}^{\ell}$. We say that an $\mathrm{R}$-linear code is free if it is a free as $R$-module.

3.1. Type and rank of a linear code. A matrix $G$ is called a generator matrix for $\mathscr{C}$ if the rows of $G$ span $\mathscr{C}$ and none of them can be written as an R-linear combination of the other rows of $G$. We say that $G$ is a generator matrix in standard form if

$$
G=\left(\begin{array}{cccccc}
I_{k_{0}} & G_{0,1} & G_{0,2} & \cdots & G_{0, s-1} & G_{0, s} \\
0 & \theta I_{k_{1}} & \theta G_{1,2} & \cdots & \theta G_{1, s-1} & \theta G_{1, s} \\
\cdots & \cdots & \cdots & \cdots & \cdots & \cdots \\
0 & 0 & 0 & \cdots & \theta^{s-1} I_{k_{s-1}} & \theta^{s-1} G_{s-1, s}
\end{array}\right) U,
$$

where $U$ is a suitable permutation matrix. The $s$-tuple $\left(k_{0}, k_{1}, \cdots, k_{s-1}\right)$ is called type of $G$ and $\operatorname{rank}(G):=k_{0}+k_{1}+\cdots+k_{s-1}$ is the $\operatorname{rank}$ of $G$.

Proposition 3. (9) Proposition 3.2, Theorem 3.5]) Each $\mathrm{R}$-linear code $\mathscr{C}$ admits a generator matrix $G$ standard form. Moreover, the type is the same for any generator matrix in standard form for $\mathscr{C}$.

So the type and the rank are the invariants of $\mathscr{C}$, and henceforth we have the following definition.

Definition 3.1. Let $\mathscr{C}$ be an $\mathrm{R}$-linear code. 
(1) The type of $\mathscr{C}$ is the type of a generator matrix of $\mathscr{C}$ in standard form.

(2) The rank of $\mathscr{C}$, denoted $\operatorname{rank}_{\mathrm{R}}(\mathscr{C})$, is the rank of a generator matrix of $\mathscr{C}$ in standard form.

Obviously, any $\mathrm{R}$-linear code $\mathscr{C}$ of length $\ell$ and of type $\left(k_{0}, k_{1}, \cdots, k_{s-1}\right)$ is free if and only if the rank of $\mathscr{C}$ is $k_{0}$, and $k_{1}=k_{2}=\cdots=k_{s-1}=0$. It defines the scalar product on $\mathrm{R}^{\ell}$ by: $\underline{\mathbf{a}}^{\prime} \cdot \underline{\mathbf{b}}^{\mathrm{T}}:=\sum_{i=0}^{\ell-1} \mathbf{a}_{i} \mathbf{b}_{i}$, where $\underline{\mathbf{b}}^{\mathrm{T}}$ is the transpose of $\underline{\mathbf{b}}$. Let $\mathscr{C}$ be an $\mathrm{R}$-linear code of length $\ell$. The dual code of $\mathscr{C}$, denoted $\mathscr{C}^{\perp}$, is an $\mathrm{R}$-linear code of length $\ell$, define by: $\mathscr{C} \perp:=\left\{\underline{\mathbf{a}} \in \mathrm{R}^{\ell}: \underline{\mathbf{a}} \cdot \underline{\mathbf{b}}^{\mathrm{T}}=0\right.$ for all $\left.\underline{\mathbf{c}} \in \mathscr{C}\right\}$. A generator matrix of $\mathscr{C}^{\perp}$, is called parity-check matrix of $\mathscr{C}$.

Proposition 4. ([9, Theorem 3.10]) Let $\mathscr{C}$ be an $\mathrm{R}$-linear code of length $\ell$ and of type $\left(k_{0}, k_{1}, \cdots, k_{s-1}\right)$. Then

(1) the type of $\mathscr{C}^{\perp}$ is $\left(\ell-k, k_{s-1}, \cdots, k_{1}\right)$, where $k:=k_{0}+k_{1}+\cdots+k_{s-1}$.

(2) $|\mathscr{C}|=q^{\sum_{t=0}^{s-1}(s-t) k_{t}}$, where $|\mathscr{C}|$ denotes the number of elements of $\mathscr{C}$.

3.2. Galois closure of a linear code over a finite chain ring. Let $\mathscr{B}$ be an $\mathrm{S}$-linear codes of length $\ell$. Then

$$
\sigma(\mathscr{B}):=\left\{\left(\sigma\left(\mathbf{c}_{0}\right), \cdots, \sigma\left(\mathbf{c}_{\ell-1}\right)\right):\left(\mathbf{c}_{0}, \cdots, \mathbf{c}_{\ell-1}\right) \in \mathscr{B}\right\}
$$

is also an $\mathrm{S}$-linear codes of length $\ell$. We say that the $\mathrm{S}$-linear code $\mathscr{B}$ is called $\sigma$-invariant if $\sigma(\mathscr{B})=$ $\mathscr{B}$. The subring subcode of $\mathscr{B}$ to $\mathrm{R}$, is $\mathrm{R}$-linear code $\operatorname{Res}_{\mathrm{R}}(\mathscr{B}):=\mathscr{B} \cap \mathrm{R}^{\ell}$, and the trace code of $\mathscr{B}$ over $R$, is the $R$-linear code

$$
\operatorname{Tr}_{\mathrm{R}}^{\mathrm{S}}(\mathscr{B}):=\left\{\left(\operatorname{Tr}_{\mathrm{R}}^{\mathrm{S}}\left(\mathbf{c}_{0}\right), \cdots, \operatorname{Tr}_{\mathrm{R}}^{\mathrm{S}}\left(\mathbf{c}_{\ell-1}\right)\right):\left(\mathbf{c}_{0}, \cdots, \mathbf{c}_{\ell-1}\right) \in \mathscr{B}\right\} .
$$

It is clear that $\operatorname{Tr}_{\mathrm{R}}^{\mathrm{S}}(\sigma(\mathscr{B}))=\operatorname{Tr}_{\mathrm{R}}^{\mathrm{S}}(\mathscr{B})$. The extension code of an $\mathrm{R}$-linear code $\mathscr{C}$ to $\mathrm{S}$, is the $\mathrm{S}$-linear code $\operatorname{Ext}_{S}(\mathscr{C})$, formed by taking all combinations of codewords of $\mathscr{C}$. The following theorem generalizes Delsarte's celebrated result (see [13, Ch.7.\$8. Theorem 11.]).

Theorem 2. (17, Theorem 3]). Let $\mathscr{B}$ be an $\mathrm{S}$-linear code then $\operatorname{Tr}_{\mathrm{R}}^{\mathrm{S}}\left(\mathscr{B}^{\perp}\right)=\operatorname{Res}_{\mathrm{R}}(\mathscr{B})^{\perp}$, where $\mathscr{B}^{\perp}$ is the dual to $\mathscr{B}$ with respect to the usual scalar product, and $\operatorname{Res}_{\mathrm{R}}(\mathscr{B})^{\perp}$ is the dual of $\operatorname{Res}_{\mathrm{R}}(\mathscr{B})$ in $\mathrm{R}^{\ell}$.

Definition 3.2. Let $\mathscr{B}$ be an $\mathrm{S}$-linear code. The $\sigma$-closure of $\mathscr{B}$, is the smallest $\sigma$-invariant $\mathrm{S}$-linear code $\widetilde{B}$, containing $\mathscr{B}$.

Proposition 5. Let $\mathscr{B}$ be an $\mathrm{S}$-linear code. Then $\widetilde{\mathscr{B}}=\sum_{i=0}^{m-1} \sigma^{i}(\mathscr{B})$ and $\operatorname{Tr}_{\mathrm{R}}^{\mathrm{S}}(\mathscr{B})=\operatorname{Tr}_{\mathrm{R}}^{\mathrm{S}}(\widetilde{\mathscr{B}})$.

Proof.We have $\mathscr{B} \subseteq \widetilde{\mathscr{B}}$ and $\sigma(\widetilde{\mathscr{B}})=\widetilde{\mathscr{B}}$, by Definition 3.2 of $\widetilde{\mathscr{B}}$. So $\sigma^{i}(\mathscr{B}) \subseteq \widetilde{\mathscr{B}}$, for all $i \in\{0,1, \cdots, m-$ 1\}. Hence $\sum_{i=0}^{m-1} \sigma^{i}(\mathscr{B}) \subseteq \widetilde{\mathscr{B}}$. Since $\sigma\left(\sum_{i=0}^{m-1} \sigma^{i}(\mathscr{B})\right)=\sum_{i=0}^{m-1} \sigma^{i}(\mathscr{B})$ and $\mathscr{B} \subseteq \sum_{i=0}^{m-1} \sigma^{i}(\mathscr{B})$, as $\widetilde{\mathscr{B}}$ is the smallest $\mathrm{S}$-linear code containing $\mathscr{B}$, which is $\sigma$-invariant, it follows $\widetilde{\mathscr{B}} \subseteq \sum_{i=0}^{m-1} \sigma^{i}(\mathscr{B})$. Hence $\widetilde{\mathscr{B}}=$ $\sum_{i=0}^{m-1} \sigma^{i}(\mathscr{B})$. Thanks to [7, Proposition 1.], $\operatorname{Tr}_{\mathrm{R}}^{\mathrm{S}}(\widetilde{\mathscr{B}})=\operatorname{Tr}_{\mathrm{R}}^{\mathrm{S}}(\mathscr{B})$.

The following Theorem summarizes the obtained results in [7].

Theorem 3. Let $\mathscr{B}$ be an $\mathrm{S}$-linear code and $\sigma$ be a generator of $\operatorname{Aut}_{\mathrm{R}}(\mathrm{S})$. Then the following statements are equivalent:

(1) $\mathscr{B}$ is $\sigma$-invariant;

(2) $\operatorname{Tr}_{\mathrm{R}}^{\mathrm{S}}(\mathscr{B})=\operatorname{Res}_{\mathrm{R}}(\mathscr{B})$;

(3) $\mathscr{B}$, and $\operatorname{Res}_{\mathrm{R}}(\mathscr{B})$ have the same type.

Proof.Let $\mathscr{B}$ be an S-linear code. 
1. $\Leftrightarrow 2 .:$ Thanks to [7, Theorem 2].

$1 . \Leftrightarrow 3$.: Since any $R$-basis of $\operatorname{Res}_{R}(\mathscr{B})$ is also an $S$-basis of $\operatorname{Ext}_{S}\left(\operatorname{Res}_{R}(\mathscr{B})\right)$. Thanks to [7, Theorem 1], we deduce that $\mathscr{B}=\operatorname{Ext}_{\mathrm{S}}\left(\operatorname{Tr}_{\mathrm{R}}^{\mathrm{S}}(\mathscr{B})\right)$ if and only if $\mathscr{B}$ and $\operatorname{Res}_{\mathrm{R}}(\mathscr{B})$ have the same type.

\section{Cyclic Linear Codes OVER Finite CHAin RINGS}

Let $\ell$ be a positive integer such that $\operatorname{gcd}(q, \ell)=1$. Then the remainder $q(\bmod \ell)$ of $q$ by $\ell$, belongs to $\mathbb{Z}_{\ell}^{\times}$, the positive integer $m$ denotes the multiplicative order of $q(\bmod \ell)$. Let $\Sigma_{\ell}:=\{0,1, \cdots, \ell-1\}$ be the underling set of $\mathbb{Z}_{\ell}$.

4.1. Cyclotomic cosets. Let $u$ be a positive integer. The set of multiples of $u$ in A is

$$
u \mathrm{~A}:=\{u z(\bmod \ell): z \in \mathrm{A}\}
$$

The $q$-closure of A is $\complement_{q}(\mathrm{~A}):=\cup_{i \in \mathbb{N}} q^{i} \mathrm{~A}$.

Definition 4.1. Let $z \in \Sigma_{\ell}$. The $q$-cyclotomic coset modulo $\ell$, containing $z$, the Galois closure of $\{z\}$. We simply write $\complement_{q}(z):=\complement_{q}(\{z\})$.

It denotes by $\Re_{\ell}(q)$ the set of $q$-closure subsets of $\Sigma_{\ell}$. Obviously, the $q$-cyclotomic cosets modulo $\ell$, form a partition of $\Sigma_{\ell}$. Let $\Sigma_{\ell}(q)$ be a set of representatives of each $q$-cyclotomic cosets modulo $\ell$.

Proposition 6. [1, Proposition 5.2] We have $\left|\Sigma_{\ell}(q)\right|=\sum_{d \mid \ell} \frac{\phi(\ell)}{\operatorname{ord}(q)}$, where $\phi($.$) is the Euler totient function$ and $\operatorname{ord}_{\ell}(q):=\min \left\{i \in \mathbb{N}: q^{i+1} \equiv 1(\bmod \ell)\right\}$.

Notation 1. Let $z \in \Sigma_{\ell}$ and $\mathrm{A}$ be a subset of $\Sigma_{\ell}$ and $u \in \mathbb{N}$, with $\operatorname{gcd}(u, q)=1$.

(1) The opposite of $\mathrm{A}$ is $-\mathrm{A}:=\{\ell-z: z \in \mathrm{A}\}$.

(2) The complementary of $\mathrm{A}$ is $\overline{\mathrm{A}}:=\left\{z \in \Sigma_{\ell}: z \notin \mathrm{A}\right\}$.

(3) The dual of $\mathrm{A}$ is $\mathrm{A}^{\diamond}:=\overline{-\mathrm{A}}$.

Remark 1. Let $\mathrm{A}$ be a subset of $\Sigma_{\ell}$. Then $\complement_{q}(\overline{\mathrm{A}})=\overline{\complement_{q}(\mathrm{~A})}$ and $-\complement_{q}(\mathrm{~A})=\complement_{q}(-\mathrm{A})$. Moreover $\left(\mathrm{A}^{\diamond}\right)^{\diamond}=\mathrm{A}$.

Example 4.1. We take $\ell=20, q=3$. The $q$-cyclotomic cosets modulo $\ell$, are: $\complement_{q}(\{0\})=\{0\}, \complement_{q}(\{5\})=$ $\{5,15\}, \complement_{q}(\{10\})=\{10\}$, and

$$
\begin{array}{ll}
\complement_{q}(\{1\})=\{1,3,9,7\} ; & \complement_{q}(\{2\})=\{2,6,18,14\} ; \\
\complement_{q}(\{4\})=\{4,12,16,8\} ; & \complement_{q}(\{11\})=\{11,13,19,17\} .
\end{array}
$$

So $\Sigma_{\ell}(q)=\{0,1,2,4,5,10,11\}$. We remark that $\complement_{q}(\{-z\})=\complement_{q}(\{z\})$, for every $z \in\{0,2,4,5,10\}$. We set $\mathrm{I}:=$ $[0,10]$. We have $\mathrm{A}:=\complement_{q}(\mathrm{I})=\complement_{q}(\{0,1,2,4,5,10\}),-\mathrm{A}=\complement_{q}(\{2,4,5,10,11\})$, and $\mathrm{A}^{\diamond}:=\complement_{q}(\{1\})$.

4.2. Likewise Reed-Solomon codes over finite chain rings. Let $\mathrm{S}$ be the Galois extension of $\mathrm{R}$ of degree $m$ and $\xi$ be a generator of $\Gamma(\mathrm{S}) \backslash\{0\}$. Let $\mathrm{A}:=\left\{a_{1}, a_{2}, \cdots, a_{k}\right\}$ be a subset of $\Sigma_{\ell}$. One denotes by $\mathbf{P}(\mathrm{S} ; \mathrm{A})$, the free $\mathrm{S}$-module with $\mathrm{S}$-basis $\left\{X^{a}: a \in \mathrm{A}\right\}$. Since $m$ is the smallest positive integer with $q^{m} \equiv 1(\bmod \ell)$, we can write $\eta:=\xi^{\frac{q^{m}-1}{\ell}}$ and the multiplicative order of $\eta$ is $\ell$. The evaluation

$$
\begin{array}{clc}
\mathbf{e v}_{\eta}: \mathbf{P}(\mathrm{S} ; \mathrm{A}) & \rightarrow & \mathrm{S}^{\ell} \\
f & \mapsto\left(f(1), f(\eta), \cdots, f\left(\eta^{\ell-1}\right)\right),
\end{array}
$$

is an $\mathrm{S}$-modules monomorphism. We see that if $\mathrm{A}:=\{0,1, \cdots, k-1\}$, then for any $\ell^{\text {th }}$-primitive root of unity $\eta$ in $\Gamma(\mathrm{S})$, the $\mathrm{S}$-linear code $\mathbf{e v}_{\eta}(\mathbf{P}(\mathrm{S} ; \mathrm{A}))$ is a primitive Reed-Solomon code. For this reason, we define Likewise Reed-Solomon codes which are a family of codes defined over large finite chain rings as follows.

Definition 4.2. Let $\mathrm{A}$ be a subset of $\Sigma_{\ell}$, and $\mathrm{S}$ be a finite chain ring such that $|\Gamma(\mathrm{S})| \geq \ell$. Let $\eta \in \Gamma(\mathrm{S})$ and the multiplicative order of $\eta$ is $\ell$. The $\mathrm{S}$-submodule $\boldsymbol{e v}_{\eta}(\boldsymbol{P}(\mathrm{S} ; \mathrm{A}))$ is called likewise Reed-Solomon code over $\mathrm{S}$, with defining pair $(\eta, \mathrm{A})$. 
We remark that $\mathbf{L}_{\eta}(\mathrm{S} ; \mathrm{A}):=\mathbf{e v}_{\eta}(\mathbf{P}(\mathrm{S} ; \mathrm{A}))$ is the free $\mathrm{S}$-linear code with free $\mathrm{S}$-basis $\left\{\mathbf{e v}_{\eta}\left(X^{a}\right): a \in \mathrm{A}\right\}$, where $A$ is a subset of $\Sigma_{\ell}$. We remark that $\mathbf{L}_{\eta}(\mathrm{S} ; \emptyset)=\{\mathbf{0}\}, \mathbf{L}_{\eta}(\mathrm{S} ;\{0\})=\mathbf{1}$ and $\mathbf{L}_{\eta}\left(\mathrm{S} ; \Sigma_{\ell}\right)=\mathrm{S}^{\ell}$.

Proposition 7. Let A,B be two subsets of $\Sigma_{\ell}$. Then

(1) $\boldsymbol{L}_{\eta}(\mathrm{S} ; \mathrm{A})$ is cyclic;

(2) $\boldsymbol{L}_{\eta}(\mathrm{S} ; \mathrm{A} \cup \mathrm{B})=\boldsymbol{L}_{\eta}(\mathrm{S} ; \mathrm{A})+\boldsymbol{L}_{\eta}(\mathrm{S} ; \mathrm{B})$ and $\boldsymbol{L}_{\eta}(\mathrm{S} ; \mathrm{A} \cap \mathrm{B})=\boldsymbol{L}_{\eta}(\mathrm{S} ; \mathrm{A}) \cap \boldsymbol{L}_{\eta}(\mathrm{S} ; \mathrm{B})$.

Proof. Consider the codeword $\underline{\mathbf{c}}_{a}=\left(1, \eta^{a}, \cdots, \eta^{a(\ell-1)}\right)$. Then the shift of $\underline{\mathbf{c}}_{a}$ is $\eta^{-a} \underline{\mathbf{c}}_{a}$. Since $\mathbf{L}_{\eta}(\mathrm{S} ; \mathrm{A})$ is $\mathrm{S}$-linear, we have $\eta^{-a} \underline{\mathbf{c}}_{a} \in \mathbf{L}_{\eta}(\mathrm{S} ; \mathrm{A})$. Hence $\mathbf{L}_{\eta}(\mathrm{S} ; \mathrm{A})$ is cyclic. It is clear that $\mathbf{L}_{\eta}(\mathrm{S} ; \mathrm{A} \cup \mathrm{B}) \supseteq \mathbf{L}_{\eta}(\mathrm{S} ; \mathrm{A})+$ $\mathbf{L}_{\eta}(\mathrm{S} ; \mathrm{B})$. The set $\left\{\mathbf{e v}_{\eta}\left(X^{a}\right): a \in \mathrm{A} \cup(\mathrm{B} \backslash \mathrm{A})\right\}$ is a free $\mathrm{R}$-basis of $\mathbf{L}_{\eta}(\mathrm{S} ; \mathrm{A} \cup \mathrm{B})$ and $\mathbf{L}_{\eta}(\mathrm{S} ; \mathrm{A})+\mathbf{L}_{\eta}(\mathrm{S} ; \mathrm{B})$. Hence, $\mathbf{L}_{\eta}(\mathrm{S} ; \mathrm{A} \cup \mathrm{B})=\mathbf{L}_{\eta}(\mathrm{S} ; \mathrm{A})+\mathbf{L}_{\eta}(\mathrm{S} ; \mathrm{B})$. We leave the last equality as an exercise.

Proposition 8. Let $\mathrm{A}$ be a subset of $\Sigma_{\ell}$ and $u$ be a positive integer such that $\operatorname{gcd}(\ell, u)=1$. Then

(1) $\boldsymbol{L}_{\eta}(\mathrm{S} ; \mathrm{A})=\boldsymbol{L}_{\eta}(\mathrm{S} ; u \mathrm{~A})$;

(2) $\boldsymbol{L}_{\eta}(\mathrm{S} ; \mathrm{A})^{\perp}=\boldsymbol{L}_{\eta}\left(\mathrm{S} ; \mathrm{A}^{\diamond}\right)$;

(3) $\boldsymbol{L}_{\eta}\left(\mathrm{S} ; \complement_{q}(\mathrm{~A})\right)$ is the $\sigma$-closure of $\boldsymbol{L}_{\eta}(\mathrm{S} ; \mathrm{A})$.

Proof.Assume that $\operatorname{gcd}(\ell, u)=1$. Then $\eta$ and $\eta^{u}$ are $\ell^{\text {th }}$-primitive roots of unity. Since $\left\{\mathbf{e v}_{\eta}\left(X^{a}\right): a \in\right.$ $u \mathrm{~A}\}$ is a free $\mathrm{R}$-basis of $\mathbf{L}_{\eta^{u}}(\mathrm{~S} ; \mathrm{A})$, we have $\mathbf{L}_{\eta^{u}}(\mathrm{~S} ; \mathrm{A})=\mathbf{L}_{\eta}(\mathrm{S} ; u \mathrm{~A})$.

A free $\mathrm{S}$-basis of $\mathbf{L}_{\eta}\left(\mathrm{S} ; \mathrm{A}^{\diamond}\right)$ is $\left\{\underline{\mathbf{c}}_{a}:-a \in \overline{\mathrm{A}}\right\}$ where $\underline{\mathbf{c}}_{a}:=\left(1, \eta^{-a}, \cdots, \eta^{-a(\ell-1)}\right) \in \mathbf{L}_{\eta}\left(\mathrm{S} ; \mathrm{A}^{\diamond}\right)$. Then for all $b \in \mathrm{A}, \underline{\mathbf{c}}_{b}:=\left(1, \eta^{b}, \cdots, \eta^{b(\ell-1)}\right) \in \mathbf{L}_{\eta}(\mathrm{S} ; \mathrm{A})$, we have $\underline{\mathbf{c}}_{b} \underline{\mathbf{c}}_{a}^{\mathrm{tr}}=\sum_{j=0}^{\ell-1} \eta^{(b-a) j}$. It is easy to check that $\sum_{j=0}^{\ell-1} \eta^{i j}=0$, when $i \not \equiv 0(\bmod \ell)$. Since $0<b-a<\ell$, we have $\underline{\mathbf{c}}_{b} \underline{\mathbf{c}}_{a}^{\mathrm{tr}}=0$. So $\mathbf{L}_{\eta}\left(\mathrm{S} ; \mathrm{A}^{\diamond}\right) \subseteq \mathbf{L}_{\eta}(\mathrm{S} ; \mathrm{A})^{\perp}$. Comparison of cardinality yields $\mathbf{L}_{\eta}(\mathrm{S} ; \mathrm{A})^{\perp}=\mathbf{L}_{\eta}\left(\mathrm{S} ; \mathrm{A}^{\diamond}\right)$. Finally, $\sigma\left(\mathbf{L}_{\eta}(\mathrm{S} ; \mathrm{A})\right)=\mathbf{L}_{\eta}(\mathrm{S} ; q \mathrm{~A})$. So by Proposition 5 we have

$$
\widehat{\mathbf{L}_{\eta}(\mathrm{S} ; \mathrm{A})}=\sum_{i=0}^{m-1} \mathbf{L}_{\eta}\left(\mathrm{S} ; q^{i} \mathrm{~A}\right)=\mathbf{L}_{\eta}\left(\mathrm{S} ; \bigcup_{i=0}^{m-1} q^{i} \mathrm{~A}\right) \text {. }
$$

Since $\complement_{q}(\mathrm{~A})=\bigcup_{i=0}^{m-1} q^{i} \mathrm{~A}$, we obtain $\widehat{\mathbf{L}_{\eta}(\mathrm{S} ; \mathrm{A})}=\mathbf{L}_{\eta}\left(\mathrm{S} ; \complement_{q}(\mathrm{~A})\right)$.

4.3. Trace representation of free cyclic linear codes. We introduce the map trace-evaluation $\operatorname{Tr}_{\mathrm{R}}^{\mathrm{S}} \circ \mathbf{e v}_{\eta}$ : $\mathbf{P}_{\eta}(\mathrm{S} ; \mathrm{A}) \rightarrow \mathrm{R}^{\ell}$, defined by:

$$
\operatorname{Tr}_{\mathrm{R}}^{\mathrm{S}} \circ \mathbf{e v}_{\eta}\left(X^{a}\right):=\operatorname{Tr}_{\mathrm{R}}^{\mathrm{S}}\left(1, \eta^{a}, \cdots, \eta^{a(\ell-1)}\right),
$$

for all $a \in \mathrm{A}$. In the sequel, we write: $\mathbf{C}_{\eta}(\mathrm{R} ; \mathrm{A}):=\operatorname{Tr}_{\mathrm{R}}^{\mathrm{S}}\left(\mathbf{L}_{\eta}(\mathrm{S} ; \mathrm{A})\right)$, and $\mathbf{C}_{\eta}(\mathrm{R} ; \mathrm{A})$ is a free cyclic R-linear code of length $\ell$. The immediate proprieties of trace representation of free cyclic linear codes over finite chain ring are given in the following.

Proposition 9. Let A,B be two empty subsets of $\Sigma_{\ell}$. Then

(1) $\boldsymbol{C}_{\eta}(\mathrm{R} ; \mathrm{A})=\boldsymbol{C}_{\eta}\left(\mathrm{R} ; \complement_{q}(\mathrm{~A})\right)$;

(2) $\operatorname{rank}_{\mathrm{S}}\left(\boldsymbol{L}_{\eta}\left(\mathrm{S} ; \complement_{q}(\mathrm{~A})\right)\right)=\left|\complement_{q}(\mathrm{~A})\right|$ and $\boldsymbol{C}_{\eta}(\mathrm{R} ; \mathrm{A})^{\perp}=\boldsymbol{C}_{\eta}\left(\mathrm{R} ; \mathrm{A}^{\diamond}\right)$;

(3) $\boldsymbol{C}_{\eta}(\mathrm{S} ; \mathrm{A} \cup \mathrm{B})=\boldsymbol{C}_{\eta}(\mathrm{S} ; \mathrm{A})+\boldsymbol{C}_{\eta}(\mathrm{S} ; \mathrm{B})$ and $\boldsymbol{C}_{\eta}(\mathrm{S} ; \mathrm{A} \cap \mathrm{B})=\boldsymbol{C}_{\eta}(\mathrm{S} ; \mathrm{A}) \cap \boldsymbol{C}_{\eta}(\mathrm{S} ; \mathrm{B})$.

Proof.Let A,B be two subsets of $\Sigma_{\ell}$.

(1) From Proposition $5, \mathbf{C}_{\eta}(\mathrm{R} ; \mathrm{A})=\operatorname{Tr}\left(\mathbf{L}_{\eta}(\mathrm{S} ; \mathrm{A})\right)=\operatorname{Tr}\left(\mathbf{L}_{\eta}\left(\mathrm{S} ; \complement_{q}(\mathrm{~A})\right)\right)=\mathbf{C}_{\eta}\left(\mathrm{R} ; \complement_{q}(\mathrm{~A})\right)$.

(2) Theorem 3(3) yields $\mathbf{C}_{\eta}(\mathrm{R} ; \mathrm{A})=\operatorname{Tr}\left(\mathbf{L}_{\eta}\left(\mathrm{S} ; \complement_{q}(\mathrm{~A})\right)\right)=\operatorname{Res}_{\mathrm{R}}\left(\mathbf{L}_{\eta}\left(\mathrm{S} ; \complement_{q}(\mathrm{~A})\right)\right)$. So

$$
\operatorname{rank}_{\mathrm{R}}\left(\mathbf{C}_{\eta}(\mathrm{R} ; \mathrm{A})\right)=\operatorname{rank}_{\mathrm{S}}\left(\mathbf{L}_{\eta}\left(\mathrm{S} ; \complement_{q}(\mathrm{~A})\right)\right)=\left|\complement_{q}(\mathrm{~A})\right| .
$$

From Proposition $8, \mathbf{C}_{\eta}(\mathrm{R} ; \mathrm{A})^{\perp}=\mathbf{C}_{\eta}\left(\mathrm{R} ; \mathrm{A}^{\diamond}\right)$.

The following theorem gives the number of cyclic codes and free cyclic codes over finite chain rings. 
Lemma 2. [1, Theorem 5.1] Let $\mathrm{R}$ be a finite chain ring of invariants $(q, s)$. Then the following holds:

(1) the number of cyclic $\mathrm{R}$-linear codes of length $\ell$, is equal to $(s+1)^{\left|\Sigma_{\ell}(q)\right|}$,

(2) the number of free cyclic $R$-linear codes of length $\ell$, is equal to $2^{\left|\Sigma_{\ell}(q)\right|}$.

Lemma 3. Let $\mathrm{R}$ be a finite chain ring of invariants $(q, s)$ and $\mathrm{S}$ be the Galois extension of $\mathrm{R}$ of degree $m$. Let $z \in \Sigma_{\ell}$. Set $\mathrm{S}=\mathrm{R}[\xi], m_{z}:=\left|\mathbb{C}_{q}(z)\right|, \eta:=\xi^{\frac{q^{m}-1}{\ell}}$. and $\zeta:=\eta^{-z}$. Then the map

$$
\begin{aligned}
\psi_{z}: \mathrm{R}\left[\xi^{m_{z}}\right] & \longrightarrow \boldsymbol{C}_{\eta}(\mathrm{R} ;\{z\}) \\
\boldsymbol{a} & \longmapsto \operatorname{Tr}_{\mathrm{R}}^{\mathrm{S}}\left(\mathrm{ev}_{\eta}\left(\boldsymbol{a} X^{z}\right)\right)
\end{aligned}
$$

is an $\mathrm{R}$-module isomorphism. Further $\mathrm{R}\left[\xi^{m_{z}}\right]$ is the Galois extension of $\mathrm{R}$ of degree $m_{z}$ and $\psi_{z} \circ t_{\zeta}=$ $\tau_{1} \circ \psi_{z}$, where $t_{\zeta}(\boldsymbol{a})=\boldsymbol{a} \zeta$, for all $\boldsymbol{a} \in \mathrm{R}[\eta]$.

Proof. It is clear that $a \in \operatorname{Ker}\left(\psi_{z}\right)$ if and only if $a \in \mathrm{R}\left[\xi^{m_{z}}\right]^{\perp_{\operatorname{Tr}} \cap \mathrm{R}}\left[\xi^{m_{z}}\right]$, where duality $\perp_{\operatorname{Tr}}$ is with respect to trace form. As the trace bilinear form is nondegenerate, we have $\mathrm{S}=\mathrm{R}\left[\xi^{m_{z}}\right]^{\perp_{\mathrm{Tr}}} \oplus \mathrm{R}\left[\xi^{m_{z}}\right]$ and $\operatorname{Ker}\left(\psi_{z}\right)=\{0\}$. Hence $\psi_{z}$ is an $\mathrm{R}$-module monomorphism. We remark that, $\mathbf{C}_{\eta}(\mathrm{R} ;\{z\})$ is cyclic, if and only if $\psi_{z} \circ t_{\zeta}=\tau_{1} \circ \psi_{z}$, for all $a \in \mathrm{R}[\eta]$. Finally, we have $\mathrm{S}=\mathrm{R}[\xi]$, so $\mathrm{R}\left[\xi^{m_{z}}\right]$ is the Galois extension of $\mathrm{R}$ of degree $m_{z}$. Hence, $\psi_{z}$ is an $\mathrm{R}$-module isomorphism.

Definition 4.3. A non trivial cyclic $\mathrm{R}$-linear code $\mathscr{C}$ is said to be irreducible, if for all $\mathrm{R}$-linear cyclic subcodes $\mathscr{C}_{1}$ and $\mathscr{C}_{2}$ of $\mathscr{C}$, such that, $\mathscr{C}=\mathscr{C}_{1} \oplus \mathscr{C}_{2}$, implies $\mathscr{C}_{1}=\{\boldsymbol{0}\}$ or $\mathscr{C}_{2}=\{\boldsymbol{0}\}$.

Proposition 10. The irreducible cyclic $\mathrm{R}$-linear codes are precisely $\theta^{t} \boldsymbol{C}_{\eta}(\mathrm{R} ;\{z\}) s$, where $t \in\{0,1, \cdots, s-1\}$ and $z \in \Sigma_{\ell}(q)$.

Proof.By Lemma 3, the cyclic R-linear code $\left.\mathbf{C}_{\eta}(\mathrm{R} ;\{z\})\right)$ and all the $\mathrm{R}$-linear cyclic subcodes are irreducible. Let $\mathscr{C}$ be an irreducible cyclic $\mathrm{R}$-linear code. Then the $\mathrm{R}$-linear code uot $_{s-1}(\mathscr{C}):=$ $\left\{\mathbf{c} \in \mathrm{R}^{\ell}: \theta^{s-1} \mathbf{c} \in \mathscr{C}\right\}$ is cyclic and free, and so $\operatorname{Quot}_{s-1}(\mathscr{C})=\mathbf{C}_{\eta}(\mathrm{R} ; \mathrm{A})$ for some $\mathrm{A} \subset \Sigma_{\ell}(q)$ and $\mathrm{A} \neq \emptyset$. Assume that $|A|>1$. Then $\mathbf{C}_{\eta}(R ; A)=\mathbf{C}_{\eta}\left(R ; A_{1}\right) \oplus \mathbf{C}_{\eta}\left(R ; A_{2}\right)$ where $A_{1} \cap A_{2}=\emptyset, A_{1} \neq \emptyset$ and $A_{2} \neq \emptyset$. We have $\mathscr{C} \cap \mathbf{C}_{\eta}\left(\mathrm{R} ; \mathrm{A}_{1}\right) \neq\{\mathbf{0}\}$ and $\mathscr{C} \cap \mathbf{C}_{\eta}\left(\mathrm{R} ; \mathrm{A}_{2}\right) \neq\{\mathbf{0}\}$. Therefore $\mathscr{C}=\left(\mathscr{C} \cap \mathbf{C}_{\eta}\left(\mathrm{R} ; \mathrm{A}_{1}\right)\right) \oplus\left(\mathscr{C} \cap \mathbf{C}_{\eta}\left(\mathrm{R} ; \mathrm{A}_{2}\right)\right)$. It is impossible, because $\mathscr{C}$ be an irreducible. So $|\mathrm{A}|=1$. Now, $\mathscr{C} \subseteq \mathbf{C}_{\eta}(\mathrm{R} ;\{z\})$, it follows that $\mathscr{C}=\theta^{t} \mathbf{C}_{\eta}(\mathrm{R} ;\{z\})$, for some $t \in\{0,1, \cdots, s-1\}$.

We set $\Sigma_{\ell}(q)$ a set of representatives of each $q$-cyclotomic cosets modulo $\ell$. An $(q, s)$-cyclotomic partition modulo $\ell$, is the $(s+1)$-tuple $\left(\mathrm{A}_{0}, \mathrm{~A}_{1}, \cdots, \mathrm{A}_{s}\right)$ with the property $\mathrm{A}_{t}=\complement_{q}\left(\lambda^{-1}(\{t\})\right)$, where $\lambda$ : $\Sigma_{\ell}(q) \rightarrow\{0,1, \cdots, s\}$ is a map. Denoted by

$$
\Re_{\ell}(q, s):=\left\{\left(\mathrm{A}_{0}, \mathrm{~A}_{1}, \cdots, \mathrm{A}_{s}\right):\left(\exists \lambda \in\{0,1, \cdots, s\}^{\Sigma_{\ell}(q)}\right)\left(\mathrm{A}_{t}=\lambda^{-1}(\{t\})\right)\right\}
$$

the set of $(q, s)$-cyclotomic partitions modulo $\ell$, and $\mathrm{Cy}(\mathrm{R}, \ell)$ the set of cyclic $\mathrm{R}$-linear codes of length $\ell$. We have $\left|\Re_{\ell}(q, s)\right|=(s+1)^{\left|\Sigma_{\ell}(q)\right|}$.

Example 4.2. We take $\ell=20, q=3$ and $s=2$. Then $\left|\Sigma_{\ell}(q)\right|=13$ and $\left|\Re_{\ell}(q, s)\right|=3^{7}$. An $(q, s)$-cyclotomic partition modulo $\ell$, is $\underline{\mathrm{A}}:=\left(\complement_{q}(\{0,1,2\}), \complement_{q}(\{5,11\}), \complement_{q}(\{4,10\})\right)$.

Theorem 4. Any cyclic $\mathrm{R}$-linear code $\mathscr{C}$ there exists a unique $\mathrm{A}:=\left(\mathrm{A}_{0}, \mathrm{~A}_{1}, \cdots, \mathrm{A}_{s}\right) \in \Re_{\ell}(q, s)$ such that $\mathscr{C}=\boldsymbol{C}_{\mathrm{R}}(\mathrm{A})$ and $\boldsymbol{C}_{\mathrm{R}}(\mathrm{A})=\bigoplus_{t=0}^{s-1} \theta^{t} \boldsymbol{C}_{\eta}\left(\mathrm{R} ; \mathrm{A}_{t}\right)$. Moreover, the type of $\boldsymbol{C}_{\mathrm{R}}(\underline{\mathrm{A}})$ is

$$
\left(\left|\mathbb{C}_{q}\left(\mathrm{~A}_{0}\right)\right|,\left|\mathbb{C}_{q}\left(\mathrm{~A}_{1}\right)\right|, \cdots,\left|\mathbb{C}_{q}\left(\mathrm{~A}_{s-1}\right)\right|\right)
$$

for some $\underline{\mathrm{A}}:=\left(\mathrm{A}_{0}, \mathrm{~A}_{1}, \cdots, \mathrm{A}_{s}\right) \in \Re_{\ell}(q, s)$.

Proof.Let $\mathscr{C}$ be an cyclic $\mathrm{R}$-linear code of length $\ell$. From Proposition 9 , we have $\mathrm{R}^{\ell}=\underset{z \in \Sigma_{\ell}(q)}{\bigoplus} \mathrm{C}_{\eta}(\mathrm{R} ;\{z\})$ and $\mathbf{C}_{\eta}(\mathrm{R} ;\{z\})$ 's are free irreducible cyclic $\mathrm{R}$-linear codes. Therefore $\mathscr{C}=\underset{z \in \Sigma_{\ell}(q)}{\bigoplus} \mathscr{C}_{z}$, where $\mathscr{C}_{z}=$ 
$\mathrm{C}_{\eta}(\mathrm{R} ;\{z\}) \cap \mathscr{C}$. From Proposition $10, \mathscr{C}_{z}=\theta^{t_{z}} \mathbf{C}_{\eta}(\mathrm{R} ;\{z\})$, where $t_{z} \in\{0,1, \cdots, s\}$. Hence

$$
\mathscr{C}=\bigoplus_{z \in \Sigma_{\ell}(q)} \theta^{t_{z}} \mathbf{C}_{\eta}(\mathrm{R} ;\{z\})=\bigoplus_{t=0}^{s-1} \theta^{t} \mathbf{C}_{\eta}\left(\mathrm{R} ; \mathrm{A}_{t}\right),
$$

where $\mathrm{A}_{t}=\left\{z \in \Sigma_{\ell}: t_{z}=t\right\}$. Since $\left|\Re_{\ell}(q, s)\right|=(s+1)^{\left|\Sigma_{\ell}(q)\right|}$, by Theorem 2 the uniqueness of $\underline{\mathrm{A}}:=$ $\left(\mathrm{A}_{0}, \mathrm{~A}_{1}, \cdots, \mathrm{A}_{s}\right) \in \mathfrak{\Re}_{\ell}(q, s)$ such that $\mathscr{C}=\mathbf{C}_{\mathrm{R}}(\mathrm{A})$ is guaranteed.

Moreover, for every $t \in\{0,1, \cdots, s-1\}$, the cyclic $\mathrm{R}$-linear code $\mathbf{C}_{\eta}\left(\mathrm{R} ; \mathrm{A}_{t}\right)$ is free and $\operatorname{rank}_{\mathrm{R}}\left(\mathbf{C}_{\eta}\left(\mathrm{R} ; \mathrm{A}_{t}\right)\right)=\left|\complement_{q}\left(\mathrm{~A}_{t}\right)\right|$. Since the direct sum $\bigoplus_{t=0}^{s-1} \theta^{t} \mathbf{C}_{\eta}\left(\mathrm{R} ; \mathrm{A}_{t}\right)$ gives the type of $\mathbf{C}_{\mathrm{R}}(\underline{\mathrm{A}})$, the type of $\mathbf{C}_{\mathrm{R}}(\underline{\mathrm{A}})$ is $\left(k_{0}, k_{1}, \cdots, k_{s-1}\right)$, where $k_{t}:=\left|\complement_{q}\left(\mathrm{~A}_{t}\right)\right|$, for every $t \in\{0,1, \cdots, s-1\}$.

Proposition 11. Let $\underline{\mathrm{A}}:=\left(\mathrm{A}_{0}, \mathrm{~A}_{1}, \cdots, \mathrm{A}_{s}\right) \in \Re_{\ell}(q, s)$ and $t \in\{0,1, \cdots, s-1\}$. Then $\boldsymbol{C}_{\mathrm{R}}(\underline{\mathrm{A}})^{\perp}=\boldsymbol{C}_{\mathrm{R}}\left(\underline{\mathrm{A}}^{\widetilde{\widetilde{y}}}\right)$, where $\underline{A}^{\widetilde{\gamma}}:=\left(-\mathrm{A}_{s},-\mathrm{A}_{s-1}, \cdots,-\mathrm{A}_{1},-\mathrm{A}_{0}\right)$.

Proof.Let $\underline{\mathrm{A}}:=\left(\mathrm{A}_{0}, \mathrm{~A}_{1}, \cdots, \mathrm{A}_{s}\right) \in \Re_{\ell}(q, s)$. We have $\mathbf{C}_{\mathrm{R}}(\underline{\mathrm{A}})^{\perp} \supseteq \bigcap_{u=0}^{s-1}\left(\theta^{s-u} \mathrm{R}^{\ell}+\mathrm{C}_{\eta}\left(\mathrm{R} ; \mathrm{A}_{u}^{\diamond}\right)\right)$ and $\theta^{s-t} \mathbf{C}_{\eta}\left(\mathrm{R} ;-\mathrm{A}_{t}\right) \subseteq \bigcap_{u=0}^{s-1}\left(\theta^{s-u} \mathrm{R}^{\ell}+\mathbf{C}_{\eta}\left(\mathrm{R} ; \mathrm{A}_{u}^{\diamond}\right)\right)$, for every $t \in\{1,2, \cdots, s\}$. It follows that $\mathbf{C}_{\mathrm{R}}\left(\underline{A}^{\widetilde{\sigma}}\right) \subseteq \mathbf{C}_{\mathrm{R}}(\underline{\mathrm{A}})^{\perp}$. From Proposition 4 and Theorem $4, \mathbf{C}_{R}\left(\underline{A}^{\widetilde{\curvearrowright}}\right)$ and $\mathbf{C}_{R}(\underline{A})^{\perp}$ have the same type, we have $\mathbf{C}_{R}(\underline{A})^{\perp}=$ $\mathbf{C}_{R}\left(\underline{A}^{\widetilde{\nabla}}\right)$.

\section{CONSTACYCliC LINEAR CODES OVER A FINITE CHAIN RING}

Let $\gamma \in \mathrm{R}^{\times}$and the multiplicative order of $\gamma$ is $u$. We study the structure of contractions of cyclic $\mathrm{R}$-linear codes of length $u \ell$. In the section, the usage of the map

$$
\begin{array}{rlr}
\wp: \mathrm{R}^{\ell} & \rightarrow & \mathrm{R}^{u \ell} \\
\underline{\mathbf{c}} & \mapsto\left(\gamma^{u-1} \underline{\mathbf{c}}\left|\gamma^{u-2} \underline{\mathbf{c}}\right| \cdots|\gamma \underline{\mathbf{c}},| \underline{\mathbf{c}}\right),
\end{array}
$$

will be necessary.

Definition 5.1. Let $\mathscr{C}$ be an $\mathrm{R}$-linear code of length $u \ell$ and $\mathscr{C}:=\wp(\mathscr{K})$.

(1) The $\mathrm{R}$-linear code $\mathscr{K}$ is called the contraction of a linear code of $\mathscr{C}$.

(2) The $\mathrm{R}$-linear code $\mathscr{C}$ is called the cyclic concatenation of $\mathscr{K}$.

The contraction of a class of linear cyclic codes over finite fields have been investigated in [2]. Our contribution is the generalization of this theory of contraction of cyclic codes to finite chain rings.

Lemma 4. Let $\gamma \in \mathrm{R}^{\times}$and $u$ the multiplicative order of $\gamma$. Then the map $\wp$ is an $\mathrm{R}$-module monomorphism. Moreover $\wp \circ \tau_{\gamma}=\tau_{1} \circ \wp$.

Proof.It is clear that $\wp$ is an $\mathrm{R}$-module monomorphism. Let $\underline{\mathbf{c}}:=\left(\mathbf{c}_{0}, \cdots, \mathbf{c}_{\ell-1}\right) \in \mathrm{R}^{\ell}$ and $\tau_{1}$ the cyclic shift on $\{0,1, \cdots, u \ell-1\}$. We have:

$$
\begin{aligned}
\tau_{1}(\wp(\underline{\mathbf{c}})) & =\tau_{1}\left(\cdots\left|\gamma^{i} \mathbf{c}_{0}, \cdots, \gamma^{i} \mathbf{c}_{\ell-1}\right| \gamma^{i-1} \mathbf{c}_{0}, \cdots, \gamma^{i-1} \mathbf{c}_{\ell-1} \mid \cdots\right) \\
& =\left(\cdots\left|\gamma^{i+1} \mathbf{c}_{\ell-1}, \gamma^{i} \mathbf{c}_{0}, \cdots, \gamma^{i} \mathbf{c}_{\ell-2}\right| \gamma^{i} \mathbf{c}_{\ell-1}, \gamma^{i-1} \mathbf{c}_{0}, \cdots, \gamma^{i-1} \mathbf{c}_{\ell-2} \mid \cdots\right) \\
& =\left(\gamma^{u-1} \tau_{\gamma}(\underline{\mathbf{c}})\left|\gamma^{u-2} \tau_{\gamma}(\underline{\mathbf{c}})\right| \cdots\left|\gamma \tau_{\gamma}(\underline{\mathbf{c}})\right| \tau_{\gamma}(\underline{\mathbf{c}})\right) ; \\
& =\wp\left(\tau_{\gamma}(\underline{\mathbf{c}})\right) .
\end{aligned}
$$

Hence $\wp \circ \tau_{\gamma}=\tau_{1} \circ \wp$.

Corollary 1. Let $\gamma \in \mathrm{R}^{\times}$and $u$ the multiplicative order of $\gamma$ and $\mathscr{K}$ be an $\mathrm{R}$-linear code of length $\ell$. Then $\mathscr{K}$ is $\gamma$-constacyclic if and only if $\wp(\mathscr{K})$ is cyclic $\mathrm{R}$-linear code of length $u \ell$. Moreover, $\mathscr{K}$ and $\wp(\mathscr{K})$ have the same type. 
Proof.The map $\wp$ is an $\mathrm{R}$-module monomorphism. So $\wp(\mathscr{K})$ is $\mathrm{R}$-linear code of length $u \ell$ and $\mathscr{K}$,

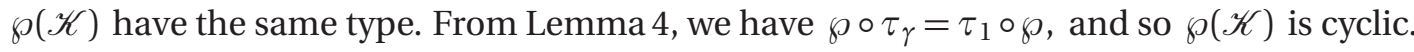

This show how to construct a cyclic $\mathrm{R}$-linear code from a constacyclic $\mathrm{R}$-linear code. Now we want to construct a constacyclic $R$-linear code from a cyclic $R$-linear code. Let $A$ be a subset of $\{0,1, \cdots, u \ell-1\}$. One denotes $\mathrm{A}(\bmod u):=\{a(\bmod u): a \in \mathrm{A}\}$.

Theorem 5. Let $u, \ell \in \mathbb{N}$ such that $\operatorname{gcd}(u \ell, q)=1$. Let $\mathrm{A}$ be a subset of $\{0,1, \cdots, u \ell-1\}$ and $\boldsymbol{C}_{\eta}(\mathrm{R} ; \mathrm{A})$ be a cyclic $\mathrm{R}$-linear code of length $u \ell$. Then $\complement_{q}(\mathrm{~A})(\bmod u)=\{\omega\}$, if and only if $\mathscr{K}:=\wp^{-1}\left(\boldsymbol{C}_{\eta}(\mathrm{R} ; \mathrm{A})\right)$ is an $\gamma$-constacyclic $\mathrm{R}$-linear code of length $\ell$, where $\gamma=\xi^{-\frac{\omega\left(q^{m}-1\right)}{u} \bmod u}$. Moreover $\mathscr{K}^{\perp}=\wp^{-1}\left(\boldsymbol{C}_{\eta}\left(\mathrm{R} ; \mathrm{A}^{\star u}\right)\right)$, where $\complement_{q}(\mathrm{~A})(\bmod u)=\{\omega\}$, and $\mathrm{A}^{\star u}:=\left\{a \in \mathrm{A}^{\diamond}: a \equiv-\omega(\bmod u)\right\}$, is an $\gamma^{-1}$-constacyclic $\mathrm{R}$-linear code of length $\ell$.

Proof.Let $m$ be the positive integer such that $q^{m} \equiv 1(\bmod u \ell)$ and $q^{m} \not \equiv 1(\bmod u \ell)$. Let $\mathrm{S}:=\mathrm{R}[\xi]$ be a Galois extension of $\mathrm{R}$ of degree $m$. We set $\beta:=\xi^{\frac{q^{m}-1}{u} \bmod u}, w:=\frac{q^{m}-1}{u \ell}$ and $\eta:=\xi^{w}$. Let $\mathrm{Z}:=\complement_{q}(\mathrm{~A})$, where $\mathrm{A}$ is a subset of $\Sigma_{\ell}$. Then $\mathbf{C}_{\eta}(\mathrm{R} ; \mathrm{Z})=\oplus_{z \in Z} \mathbf{C}_{\eta}(\mathrm{R} ;\{z\})$. It is enough to show that $\mathbf{C}_{\eta}(\mathrm{R} ;\{z\}) \subseteq \wp\left(\mathrm{R}^{\ell}\right)$, for all $z \in \mathrm{Z}$. Let $z \in \mathrm{Z}$, we set $m_{z}:=\left|\mathrm{C}_{q}(z)\right|$ and $\zeta:=\eta^{m_{z}}$. From Lemma 3 , $\mathbf{C}_{\eta}(\mathrm{R} ;\{z\})=\psi_{z}\left(\mathrm{R}\left[\xi^{m_{z}}\right]\right)=\operatorname{Tr}_{\mathrm{R}}^{\mathrm{S}}\left(e v_{\eta}\left(\mathrm{R}\left[\xi^{m_{z}}\right] X^{z}\right)\right)$. Thus for all $\underline{\mathbf{c}}:=\left(\mathbf{c}_{0}, \cdots, \mathbf{c}_{u \ell-1}\right) \in \mathbf{C}_{\eta}(\mathrm{R} ;\{z\})$, From Lemma3, exist a unique $\mathbf{a} \in \mathrm{R}\left[\xi^{m_{z}}\right]$ and such that $\underline{\mathbf{c}}=\operatorname{Tr}_{\mathrm{R}}^{\mathrm{S}}\left(e v_{\eta}\left(\mathbf{a} X^{z}\right)\right)$. Since $\mathrm{R}\left[\xi^{m_{z}}\right]$ is the Galois extension of $\mathrm{R}$ of degree $m_{z}$, then there exist a unique $\left(\mathbf{a}_{0}, \mathbf{a}_{1}, \cdots, \mathbf{a}_{m_{z}-1}\right)$ such that $\mathbf{a}:=\sum_{h=0}^{m_{z}-1} \mathbf{a}_{h} \xi^{h m_{z}} \in \mathrm{R}\left[\xi^{m_{z}}\right]$ and $\mathbf{c}_{t}:=\sum_{h=0}^{m_{z}-1} \mathbf{a}_{h} \operatorname{Tr}_{\mathrm{R}}^{\mathrm{S}}\left(\xi^{h m_{z}+w t z \bmod \ell}\right)$, for all $t \in \Sigma_{u \ell}$. From the euclidian division of $t \in\{0,1, \cdots, u \ell-1\}$, by $\ell$, there exists $(i, j) \in \Sigma_{u} \times \Sigma_{\ell}$, such that $t=i \ell+j$. We have:

$$
\begin{aligned}
\mathbf{c}_{i \ell+j} & =\sum_{h=0}^{m_{z}-1} \mathbf{a}_{h} \operatorname{Tr}_{\mathrm{R}}^{\mathrm{S}}\left(\beta^{z i} \xi^{h m_{z}+w j z \bmod \ell}\right), \text { since } \beta=\xi^{w \ell \bmod u} \text { and } \beta^{z \bmod u}=\beta^{\omega \bmod u} \\
& =\beta^{\omega i \bmod u}\left(\sum_{h=0}^{m_{z}-1} \mathbf{a}_{h} \operatorname{Tr}_{\mathrm{R}}^{\mathrm{S}}\left(\xi^{h m_{z}+w j z \bmod \ell}\right)\right), \text { since } \beta^{\omega \bmod u} \in \mathrm{R} ; \\
& =\beta^{\omega i \bmod u} \mathbf{x}_{j} \text { and } \mathbf{x}_{j}:=\sum_{h=0}^{m_{z}-1} \mathbf{a}_{h} \operatorname{Tr}_{\mathrm{R}}^{\mathrm{S}}\left(\xi^{h m_{z}+w j z \bmod \ell}\right) .
\end{aligned}
$$

Thus $\mathbf{c}:=\left(\cdots\left|\gamma^{i} \mathbf{x}_{0}, \cdots, \gamma^{i} \mathbf{x}_{\ell-1}\right| \gamma^{i-1} \mathbf{x}_{0}, \cdots, \gamma^{i-1} \mathbf{x}_{\ell-1} \mid \cdots\right)$ and $\gamma:=\beta^{-\omega}$. Hence $\mathbf{C}_{\eta}(\mathrm{R} ; \mathrm{A}) \subseteq \wp\left(\mathrm{R}^{\ell}\right)$. As $\wp \circ \tau_{\gamma}=\tau_{1} \circ \wp$, it follows that $\mathscr{K}$ is an $\gamma$-constacyclic $R$-linear code of length $\ell$. For sufficiency, it is enough to note that the above proof is reversible.

As $\mathscr{K}^{\perp}$ is an $\gamma^{-1}$-constacyclic free $\mathrm{R}$-linear code of rank $\ell-|\mathrm{A}|$, the cyclic $\mathrm{R}$-linear code which yields $\mathscr{K}^{\perp}$, by contraction must have the definition set $\mathrm{B}$ of size $|\mathrm{A}|$.

Example 5.1. Let $\mathrm{R}$ be a finite chain ring of invariants $(q, s)$ where $q=3$. We take $\ell=28, u=2$. We set $A_{1}:=\complement_{q}(\{1,7\}), A_{2}:=\complement_{q}(\{1,5,7\})$, and $A_{3}:=\complement_{q}(\{1,5,7,11\})$. We have $\complement_{q}\left(A_{i}\right)(\bmod 2)=\{1\}$. So we can set $\mathscr{K}_{i}:=\wp^{-1}\left(\boldsymbol{C}_{\eta}\left(\mathrm{R} ; \mathrm{A}_{i}\right)\right)$, where $i \in\{1,2,3\}$. Since $\mathrm{A}_{1}^{\star 2}=\mathrm{A}_{3}$ and $\mathrm{A}_{2}^{\star 2}=\mathrm{A}_{2}$, we have $\mathscr{K}_{3}=\mathscr{K}_{1}^{\perp}$ and $\mathscr{K}_{2}$ is self-dual.

The Hamming weight of an $\mathrm{R}$-linear code $\mathscr{C}$ of length $\ell$, is defined as: $\operatorname{wt}(\mathscr{C}):=$ $\min \{\operatorname{wt}(\underline{\mathbf{c}}): \mathbf{c} \in \mathscr{C} \backslash\{\mathbf{0}\}\}$, where $\operatorname{wt}(\underline{\mathbf{c}}):=\left|\left\{j \in \Sigma_{\ell}: \mathbf{c}_{j} \neq 0\right\}\right|$.

Corollary 2. Let $u, \ell \in \mathbb{N}$ such that $\operatorname{gcd}(u \ell, q)=1$. Let $\underline{\mathrm{A}}:=\left(\mathrm{A}_{0}, \mathrm{~A}_{1}, \cdots, \mathrm{A}_{s}\right) \in \Re_{u \ell}(q, s)$ and $\boldsymbol{C}_{\mathrm{R}}(\underline{\mathrm{A}})$ be a cyclic $\mathrm{R}$-linear code of length $u \ell$ such that $\bigcup_{t=0}^{s-1} \mathrm{~A}_{t}(\bmod u)=\{\omega\}$. Set $\mathscr{K}:=\wp^{-1}\left(\boldsymbol{C}_{\mathrm{R}}(\underline{\mathrm{A}})\right)$. Then

(1) $\mathscr{K}$ is an $\gamma$-constacyclic $\mathrm{R}$-linear code of length $\ell$, where $\gamma=\xi^{-\frac{\omega\left(q^{m}-1\right)}{u} \bmod u}$; 
(2) $\operatorname{wt}(\underline{\boldsymbol{c}})=u \cdot \operatorname{wt}\left(\wp^{-1}(\underline{\boldsymbol{c}})\right)$, for every $\underline{\boldsymbol{c}} \in \boldsymbol{C}_{\mathrm{R}}(\underline{\mathrm{A}})$;

(3) $\mathscr{K}^{\perp}=\wp^{-1}\left(\boldsymbol{C}_{\mathrm{R}}\left(\underline{\mathrm{A}}^{\star u}\right)\right)$, where $\underline{\mathrm{A}}^{\star u}:=\left(-\mathrm{A}_{s}^{\star u},-\mathrm{A}_{s-1}, \cdots,-\mathrm{A}_{1},-\mathrm{A}_{0}^{\triangleleft u}\right)$ with

- $\mathrm{A}_{s}^{\star u}:=\left\{a \in \mathrm{A}_{s}: a \equiv-\omega(\bmod u)\right\}$,

- $\mathrm{A}_{0}^{\triangleleft u}:=\mathrm{A}_{0} \cup\left(\mathrm{A}_{s} \backslash \mathrm{A}_{s}^{\star u}\right)$.

Example 5.2. Let $\mathrm{R}$ be a finite chain ring of invariants $(q, s)$ where $\mathrm{J}(\mathrm{R})=\mathrm{R} \theta, q=3$ and $s=2$. We take $\ell=10, u=2$. We set $\underline{A}:=\left(A_{0}, A_{1}, A_{2}\right)$, where $A_{0}:=\complement_{q}(\{1\}), A_{1}:=\complement_{q}(\{5\})$, and $A_{2}:=\complement_{q}(\{0,2,4,10,11\})$. We have $\complement_{q}\left(\mathrm{~A}_{0}\right)(\bmod 2)=\complement_{q}\left(\mathrm{~A}_{1}\right)(\bmod 2)=\{1\}$. So the contraction of the cyclic $\mathrm{R}$-linear code $C_{\mathrm{R}}(\underline{\mathrm{A}})$ of length 20 , is the self-dual negacyclic $\mathrm{R}$-linear code $\mathscr{K}:=\wp^{-1}\left(\boldsymbol{C}_{\eta}\left(\mathrm{R} ; \mathrm{A}_{0}\right)\right) \oplus \theta \wp^{-1}\left(\boldsymbol{C}_{\eta}\left(\mathrm{R} ; \mathrm{A}_{1}\right)\right)$, of length 10.

\section{CONCLUSION}

We have seen that in the case $\operatorname{gcd}(\ell,|R|)=1$, and $\gamma \in \Gamma(R)^{*}$, the class of $\gamma$-constacyclic $R$-linear codes of length $\ell$, is the same as the class of contractions of cyclic $R$-linear codes $C_{R}\left(A_{0}, A_{1}, \cdots, A_{s}\right)$ of length $u \ell$, where $u$ is the multiplicative order of $\gamma$, and each cyclic $R$-linear code $\mathbf{C}_{R}\left(\mathrm{~A}_{0}, \mathrm{~A}_{1}, \cdots, \mathrm{A}_{s}\right)$ of this class, satisfies: $\bigcup_{t=0}^{s-1} \mathrm{~A}_{t}(\bmod u)$ is a singleton.

\section{REFERENCES}

[1] Batoul A., Guenda K., Guelliver T.A., On the self-dual cyclic codes over finite chain rings, Des. Codes Cryptogr. 70(1) 347-358 (2014)

[2] Bierbrauer J., The Theory of Cyclic Codes and a Generalization to Additive Codes. Des.Codes.Cryptogr. 25(2): 189-206 (2002).

[3] Yonglin Cao, On constacyclic codes over finite chain rings, Finite Fields and Their Applications 24 (2013) 124-135.

[4] H. Dinh, S.R. López-Permouth, Cyclic and negacyclic codes over finite chain rings, IEEE Trans. Inform. Theory 50 (8) $1728-$ 1744(2004).

[5] Kai X., Zhu S., Tang Y., Some constacyclic self-dual codes over the integers modulo $2^{m}$, Finite Fields Appl. 18 (2012) $258-270$

[6] McDonald B. R., Finite Rings with Identity, Marcel Dekker, New York (1974),

[7] Martinez-Moro E., Nicolas A.P.,Rua F.,On trace codes and Galois invariance over finite commutative chain rings, Finite Fields Appl. Vol. 22, pp. 114-121 (2013).

[8] Alexandr A. Nechaev, Finite rings with applications, in: Handbook of Algebra, vol. 5, Elsevier/North-Holland, Amsterdam, 2008, pp. 213-320.

[9] Norton G.H., Sãlãgean A., On the Structure of Linear and Cyclic Codes over a Finite Chain Ring, AAECC Vol. 10, pp. 489-506, (2000).

[10] H. Tapia-Recillas, G. Vega, Some constacyclic codes over $\mathbb{Z}_{2^{k}}$ and binary quasi-cyclic codes, Discrete Appl. Math. 128 (2003) 305-316.

[11] Wolfmann J., Negacyclic and cyclic codes over $\mathbb{Z}_{4}$, IEEE Trans. Inform. Theory 45 (7) (1999) 2527-2532.

[12] Zhu S., Kai X., A class of constacyclic codes over $\mathbb{Z}_{p^{m}}$, Finite Fields Appl. 16 (2010) 243-254.

[13] McWilliams F. J. and Sloane N. J. A., The Theory of Error-Correcting Codes, North-Holland Mathematical Library, Vol.16, North-Holland Publishing Co., Amsterdam, (1977).

Department of mathematics, Faculty of Sciences, University of Yaoundé 1, Cameroon

E-mail address: alexfotue@gmail .com

Department of mathematics, Higher Teachers Training College of Yaoundé, University of Yaoundé 1 , Cameroon

E-mail address: cmouaha@yahoo.fr 